\title{
Cohomology Characterizations of Diagonal Non-Abelian Extensions of Regular Hom-Lie Algebras ${ }^{\dagger}$
}

\author{
Lina Song * and Rong Tang \\ Department of Mathematics, Jilin University, Changchun 130012, China; tangrong16@mails.jlu.edu.cn \\ * Correspondence: songln@jlu.edu.cn \\ † Research supported by NSFC (11471139) and NSF of Jilin Province (20170101050JC).
}

Received: 18 July 2017; Accepted: 29 November 2017; Published: 5 December 2017

\begin{abstract}
In this paper, first we show that under the assumption of the center of $\mathfrak{h}$ being zero, diagonal non-abelian extensions of a regular Hom-Lie algebra $\mathfrak{g}$ by a regular Hom-Lie algebra $\mathfrak{h}$ are in one-to-one correspondence with Hom-Lie algebra morphisms from $\mathfrak{g}$ to Out $(\mathfrak{h})$. Then for a general Hom-Lie algebra morphism from $\mathfrak{g}$ to $\operatorname{Out}(\mathfrak{h})$, we construct a cohomology class as the obstruction of existence of a non-abelian extension that induces the given Hom-Lie algebra morphism.
\end{abstract}

Keywords: Hom-Lie algebras; derivations; non-abelian extensions; obstruction classes

MSC: 17B40; 17B70; 18D35

\section{Introduction}

The notion of a Hom-Lie algebra was introduced by Hartwig, Larsson, and Silvestrov in [1] as part of a study of deformations of the Witt and the Virasoro algebras. In a Hom-Lie algebra, the Jacobi identity is twisted by a linear map, called the Hom-Jacobi identity. The set of $(\sigma, \sigma)$-derivations of an associative algebra and some $q$-deformations of the Witt and the Virasoro algebras have the structure of a Hom-Lie algebra [1-3]. Because of the close relation to discrete and deformed vector fields and differential calculus $[1,4,5]$, more people have started paying attention to this algebraic structure. In particular, representations and deformations of Hom-Lie algebras are studied in [6-8]; extensions of Hom-Lie algebras are studied in $[4,9,10]$. Some split regular Hom-structures are studied in $[11,12]$.

The notion of a Hom-Lie 2-algebra, which is the categorification of a Hom-Lie algebra, is given in [13]. The category of Hom-Lie 2-algebras and the category of 2-term $H L_{\infty}$-algebras are equivalent. Skeletal Hom-Lie 2-algebras can be classified by the third cohomology group of a Hom-Lie algebra. Many known Hom-structures, such as Hom-pre-Lie algebras and symplectic Hom-Lie algebras, lead to skeletal or strict Hom-Lie 2-algebras. In [14], we give the notion of a derivation of a regular Hom-Lie algebra $\left(\mathfrak{g},[\cdot, \cdot]_{\mathfrak{g}}, \phi_{\mathfrak{g}}\right)$. The set of derivations $\operatorname{Der}(\mathfrak{g})$ is a Hom-Lie subalgebra of the regular Hom-Lie algebra $\left(\mathfrak{g l}(\mathfrak{g}),[\cdot, \cdot]_{\phi_{\mathfrak{g}}}, \operatorname{Ad}_{\phi_{\mathfrak{g}}}\right)$, which is given in [15]. We constructed the derivation Hom-Lie 2-algebra $\operatorname{DER}(\mathfrak{g})$, by which we characterize non-abelian extensions of regular Hom-Lie algebras as Hom-Lie 2-algebra morphisms. More precisely, we characterize a diagonal non-abelian extension of a regular Hom-Lie algebra $\mathfrak{g}$ by a regular Hom-Lie algebra $\mathfrak{h}$ using a Hom-Lie 2-algebra morphism from $\mathfrak{g}$ to the derivation Hom-Lie 2-algebra DER(h). Associated to a non-abelian extension of a regular Hom-Lie algebra $\mathfrak{g}$ by a regular Hom-Lie algebra $\mathfrak{h}$, there is a Hom-Lie algebra morphism from $\mathfrak{g}$ to Out $(\mathfrak{h})$ naturally. However, given an arbitrary Hom-Lie algebra morphism from $\mathfrak{g}$ to Out $(\mathfrak{h})$, whether there is a non-abelian extension of $\mathfrak{g}$ by $\mathfrak{h}$ that induces the given Hom-Lie algebra morphism and what the obstruction is are not known yet. 
The aim of this paper is to solve the above problem. It turns out that the result is not totally parallel to the case of Lie algebras [16-21]. We need to add some conditions on the short exact sequence related to derivations of Hom-Lie algebras. Under these conditions, first we show that under the assumption of the center of $\mathfrak{h}$ being zero, there is a one-to-one correspondence between diagonal non-abelian extensions of $\mathfrak{g}$ by $\mathfrak{h}$ and Hom-Lie algebra morphisms from $\mathfrak{g}$ to Out $(\mathfrak{h})$. Then for the general case, we show that the obstruction of the existence of a non-abelian extension is given by an element in the third cohomology group.

The paper is organized as follows. In Section 2, we recall some basic notions of Hom-Lie algebras, representations of Hom-Lie algebras, their cohomologies and derivations of Hom-Lie algebras. In Section 3, we study non-abelian extensions of $\mathfrak{g}$ by $\mathfrak{h}$ in the case that the center of $\mathfrak{h}$ is zero. We show that if the center of $\mathfrak{h}$ is zero and the short exact sequence related to derivations of Hom-Lie algebras is also diagonal, then diagonal non-abelian extensions of $\mathfrak{g}$ by $\mathfrak{h}$ correspond bijectively to Hom-Lie algebra morphisms from $\mathfrak{g}$ to Out $(\mathfrak{h})$ (Theorem 2 ). In Section 4 , we give a cohomology characterization of the existence of general non-abelian extensions of $\mathfrak{g}$ by $\mathfrak{h}$. We show that the obstruction of the existence of a diagonal non-abelian extension of $\mathfrak{g}$ by $\mathfrak{h}$ that induces a given Hom-Lie algebra morphism from $\mathfrak{g}$ to Out $(\mathfrak{h})$ is given by a cohomology class in $\mathcal{H}^{3}(\mathfrak{g} ; \operatorname{Cen}(\mathfrak{h}))$ (Theorem 3). Moreover, isomorphism classes of diagonal non-abelian extensions of $\mathfrak{g}$ by $\mathfrak{h}$ are parameterized by $\mathcal{H}^{2}(\mathfrak{g} ; \operatorname{Cen}(\mathfrak{h}))$ (Theorem 4$)$. In Section 5, we give a conclusion of the paper.

\section{Preliminaries}

In this paper, we work over an algebraically closed field $\mathbb{K}$ of characteristic 0 , and all the vector spaces are over $\mathbb{K}$. We only work on finite-dimensional vector spaces.

\subsection{Representations, Cohomologies and Derivations of Hom-Lie Algebras}

\section{Definition 1.}

(i) A (multiplicative) Hom-Lie algebra is a triple $\left(\mathfrak{g},[\cdot, \cdot]_{\mathfrak{g}}, \phi_{\mathfrak{g}}\right)$ consisting of a vector space $\mathfrak{g}$, a skew-symmetric bilinear map (bracket) $[\cdot, \cdot]_{\mathfrak{g}}: \wedge^{2} \mathfrak{g} \longrightarrow \mathfrak{g}$ and a linear map $\phi_{\mathfrak{g}}: \mathfrak{g} \rightarrow \mathfrak{g}$ preserving the bracket, such that the following Hom-Jacobi identity with respect to $\phi_{\mathfrak{g}}$ is satisfied:

$$
\left[\phi_{\mathfrak{g}}(x),[y, z]_{\mathfrak{g}}\right]_{\mathfrak{g}}+\left[\phi_{\mathfrak{g}}(y),[z, x]_{\mathfrak{g}}\right]_{\mathfrak{g}}+\left[\phi_{\mathfrak{g}}(z),[x, y]_{\mathfrak{g}}\right]_{\mathfrak{g}}=0
$$

(ii) A Hom-Lie algebra is called a regular Hom-Lie algebra if $\phi_{\mathfrak{g}}$ is an algebra automorphism.

(iii) The center Cen $(\mathfrak{g})$ of a regular Hom-Lie algebra $\left(\mathfrak{g},[\cdot, \cdot]_{\mathfrak{g}}, \phi_{\mathfrak{g}}\right)$ is defined by

$$
\operatorname{Cen}(\mathfrak{g})=\left\{x \in \mathfrak{g} \mid[x, y]_{\mathfrak{g}}=0, \forall y \in \mathfrak{g}\right\}
$$

Remark 1. The center of a Hom-Lie algebra $\left(\mathfrak{g},[\cdot, \cdot]_{\mathfrak{g}}, \phi_{\mathfrak{g}}\right)$ (not necessarily regular) is usually defined by Equation (2); see [9] (Definition 2.13). However, for $x \in \operatorname{Cen}(\mathfrak{g}), \phi_{\mathfrak{g}}(x)$ may not be in $\operatorname{Cen}(\mathfrak{g})$. This is a conflict with the definition of a subalgebra of a Hom-Lie algebra, for which one requires that the subspace is closed with respect to both $[\cdot, \cdot]_{\mathfrak{g}}$ and $\phi_{\mathfrak{g}}$. We note that for a regular Hom-Lie algebra, if $x \in \mathfrak{g}$ is such that $[x, y]=0$ for all $y \in \mathfrak{g}$, then $\phi_{\mathfrak{g}}(x)$ also satisfies this property. This follows from

$$
\left[\phi_{\mathfrak{g}}(x), y\right]_{\mathfrak{g}}=\left[\phi_{\mathfrak{g}}(x), \phi_{\mathfrak{g}} \circ \phi_{\mathfrak{g}}^{-1}(y)\right]_{\mathfrak{g}}=\phi_{\mathfrak{g}}\left[x, \phi_{\mathfrak{g}}^{-1}(y)\right]_{\mathfrak{g}}=0, \quad \forall y \in \mathfrak{g}
$$

Thus, we suggest that for a general Hom-Lie algebra, one should define its center by

$$
\operatorname{Cen}(\mathfrak{g})=\left\{x \in \mathfrak{g} \mid\left[\phi_{\mathfrak{g}}^{k}(x), y\right]_{\mathfrak{g}}=0, \forall y \in \mathfrak{g}, k \in \mathbb{N}\right\}
$$

In the sequel, we always assume that $\phi_{\mathfrak{g}}$ is an algebra automorphism. That is, in this paper, all the Hom-Lie algebras are assumed to be regular Hom-Lie algebras despite that some results also hold for general Hom-Lie algebras. 
Example 1. Let $V$ be a vector space and $\beta \in G L(V)$. Define a skew-symmetric bilinear bracket operation $[\cdot, \cdot]_{\beta}: \wedge^{2} \mathfrak{g l}(V) \longrightarrow \mathfrak{g l}(V)$ by

$$
[A, B]_{\beta}=\beta \circ A \circ \beta^{-1} \circ B \circ \beta^{-1}-\beta \circ B \circ \beta^{-1} \circ A \circ \beta^{-1}, \quad \forall A, B \in \mathfrak{g l}(V)
$$

Denote by $\operatorname{Ad}_{\beta}: \mathfrak{g l}(V) \rightarrow \mathfrak{g l}(V)$ the adjoint action on $\mathfrak{g l}(V)$, that is,

$$
\operatorname{Ad}_{\beta}(A)=\beta \circ A \circ \beta^{-1}
$$

Then $\left(\mathfrak{g r}(V),[\cdot, \cdot]_{\beta}, \operatorname{Ad}_{\beta}\right)$ is a regular Hom-Lie algebra. See [15] for more details.

Definition 2. A morphism of Hom-Lie algebras $f:\left(\mathfrak{g},[\cdot, \cdot]_{\mathfrak{g}}, \phi_{\mathfrak{g}}\right) \rightarrow\left(\mathfrak{h},[\cdot, \cdot]_{\mathfrak{h}}, \phi_{\mathfrak{h}}\right)$ is a linear map $f: \mathfrak{g} \rightarrow \mathfrak{h}$ such that

$$
\begin{aligned}
f[x, y]_{\mathfrak{g}} & =[f(x), f(y)]_{\mathfrak{h}}, \quad \forall x, y \in \mathfrak{g} \\
f \circ \phi_{\mathfrak{g}} & =\phi_{\mathfrak{h}} \circ f
\end{aligned}
$$

Definition 3. A representation of a Hom-Lie algebra $\left(\mathfrak{g},[\cdot, \cdot]_{\mathfrak{g}}, \phi_{\mathfrak{g}}\right)$ on a vector space $V$ with respect to $\beta \in \mathfrak{g l}(V)$ is a linear map $\rho: \mathfrak{g} \rightarrow \mathfrak{g l}(V)$ such that for all $x, y \in \mathfrak{g}$, the following equalities are satisfied:

$$
\begin{aligned}
\rho\left(\phi_{\mathfrak{g}}(x)\right) \circ \beta & =\beta \circ \rho(x) \\
\rho\left([x, y]_{\mathfrak{g}}\right) \circ \beta & =\rho\left(\phi_{\mathfrak{g}}(x)\right) \circ \rho(y)-\rho\left(\phi_{\mathfrak{g}}(y)\right) \circ \rho(x)
\end{aligned}
$$

We denote a representation by $(\rho, V, \beta)$.

Theorem 1 ([15], (Theorem 4.2)). Let $\left(\mathfrak{g},[\cdot, \cdot]_{\mathfrak{g}}, \phi_{\mathfrak{g}}\right)$ be a Hom-Lie algebra, $V$ be a vector space, and $\beta \in G L(V)$. Then $\rho: \mathfrak{g} \rightarrow \mathfrak{g l}(V)$ is a representation of $\left(\mathfrak{g},[\cdot, \cdot]_{\mathfrak{g}}, \phi_{\mathfrak{g}}\right)$ on $V$ with respect to $\beta$ if and only if $\rho:\left(\mathfrak{g},[\cdot, \cdot]_{\mathfrak{g}}, \phi_{\mathfrak{g}}\right) \rightarrow\left(\mathfrak{g l}(V),[\cdot, \cdot]_{\beta}, \operatorname{Ad}_{\beta}\right)$ is a morphism of Hom-Lie algebras.

For all $x \in \mathfrak{g}$, we define $\operatorname{ad}_{x}: \mathfrak{g} \rightarrow \mathfrak{g}$ by

$$
\operatorname{ad}_{x}(y)=[x, y]_{\mathfrak{g}}, \quad \forall y \in \mathfrak{g}
$$

Then ad $: \mathfrak{g} \longrightarrow \mathfrak{g l}(\mathfrak{g})$ is a representation of the Hom-Lie algebra $\left(\mathfrak{g},[\cdot, \cdot]_{\mathfrak{g}}, \phi_{\mathfrak{g}}\right)$ on $\mathfrak{g}$ with respect to $\phi_{\mathfrak{g}}$, which is called the adjoint representation.

Let $(\rho, V, \beta)$ be a representation. We define the set of $k$-Hom-cochains by

$$
C_{\phi_{\mathfrak{g}}, \beta}^{k}(\mathfrak{g} ; V) \triangleq\left\{f \in C^{k}(\mathfrak{g} ; V) \mid \beta \circ \phi_{\mathfrak{g}}=\phi_{\mathfrak{g}} \circ \beta^{\otimes k}\right\}
$$

For $k \geq 1$, we define the coboundary operator $\mathrm{d}_{\rho}: C_{\phi_{\mathfrak{g}, \beta}}^{k}(\mathfrak{g} ; V) \rightarrow C_{\phi_{\mathfrak{g}, \beta}, \beta}^{k+1}(\mathfrak{g} ; V)$ by

$$
\begin{aligned}
\left(\mathrm{d}_{\rho} f\right)\left(x_{1}, \cdots, x_{k+1}\right)= & \sum_{i=1}^{k+1}(-1)^{i+1} \rho\left(\phi_{\mathfrak{g}}^{k-1}\left(x_{i}\right)\right)\left(f\left(x_{1}, \cdots, \widehat{x_{i}}, \cdots, x_{k+1}\right)\right) \\
& +\sum_{i<j}(-1)^{i+j} f\left(\left[x_{i}, x_{j}\right]_{\mathfrak{g}}, \phi_{\mathfrak{g}}\left(x_{1}\right), \cdots, \widehat{\phi_{\mathfrak{g}}\left(x_{i}\right)}, \cdots, \widehat{\phi_{\mathfrak{g}}\left(x_{j}\right)}, \cdots, \phi_{\mathfrak{g}}\left(x_{k+1}\right)\right)
\end{aligned}
$$

where $\mathrm{d}_{\rho} \circ \mathrm{d}_{\rho}=0$ is proved in [8]. Denote by $\mathcal{Z}^{k}(\mathfrak{g} ; \rho)$ and $\mathcal{B}^{k}(\mathfrak{g} ; \rho)$ the sets of $k$-cocycles and $k$-coboundaries, respectively. We define the $k$ th cohomology group $\mathcal{H}^{k}(\mathfrak{g} ; \rho)$ to be $\mathcal{Z}^{k}(\mathfrak{g} ; \rho) / \mathcal{B}^{k}(\mathfrak{g} ; \rho)$. See also [6] for more details about such cochain and coboundary setups. 
Definition 4 ([14], (Definition 3.1)). A linear map D : $\mathfrak{g} \rightarrow \mathfrak{g}$ is called a derivation of a Hom-Lie algebra $\left(\mathfrak{g},[\cdot, \cdot]_{\mathfrak{g}}, \phi_{\mathfrak{g}}\right)$ if

$$
D[x, y]_{\mathfrak{g}}=\left[\phi_{\mathfrak{g}}(x),\left(\operatorname{Ad}_{\phi_{\mathfrak{g}}^{-1}} D\right)(y)\right]_{\mathfrak{g}}+\left[\left(\operatorname{Ad}_{\phi_{\mathfrak{g}}^{-1}} D\right)(x), \phi_{\mathfrak{g}}(y)\right]_{\mathfrak{g}}, \quad \forall x, y \in \mathfrak{g}
$$

Denote by $\operatorname{Der}(\mathfrak{g})$ the set of derivations of the Hom-Lie algebra $\left(\mathfrak{g},[\cdot, \cdot]_{\mathfrak{g}}, \phi_{\mathfrak{g}}\right)$. Then we obtain that $\left(\operatorname{Der}(\mathfrak{g}),[\cdot, \cdot]_{\phi_{\mathfrak{g}}}, \operatorname{Ad}_{\phi_{\mathfrak{g}}}\right)$ is a Hom-Lie algebra, which is a subalgebra of the Hom-Lie algebra $\left(\mathfrak{g l}(\mathfrak{g}),[\cdot, \cdot]_{\phi_{\mathfrak{g}}}, \operatorname{Ad}_{\phi_{\mathfrak{g}}}\right)$.

Remark 2. The above definition of a derivation of a Hom-Lie algebra is more general than that given in [8]. Under the condition $D \circ \phi_{\mathfrak{g}}=\phi_{\mathfrak{g}} \circ D$, the above definition is the same as the $\alpha$-derivation given in [8]. See Remark 3.2 in [14] for more details.

For all $x \in \mathfrak{g}, \operatorname{ad}_{x}$ is a derivation of the Hom-Lie algebra $\left(\mathfrak{g},[\cdot, \cdot]_{\mathfrak{g}}, \phi_{\mathfrak{g}}\right)$, which we call an inner derivation. See [14] for more details. Denote by $\operatorname{Inn}(\mathfrak{g})$ the set of inner derivations of the Hom-Lie algebra $\left(\mathfrak{g},[\cdot, \cdot]_{\mathfrak{g}}, \phi_{\mathfrak{g}}\right)$, that is,

$$
\operatorname{lnn}(\mathfrak{g})=\left\{\operatorname{ad}_{x} \mid x \in \mathfrak{g}\right\}
$$

Lemma $1\left([14],\left(\right.\right.$ Lemma 3.6)). Let $\left(\mathfrak{g},[\cdot, \cdot]_{\mathfrak{g}}, \phi_{\mathfrak{g}}\right)$ be a Hom-Lie algebra. For all $x \in \mathfrak{g}$ and $D \in \operatorname{Der}(\mathfrak{g})$, we have

$$
\operatorname{Ad}_{\phi_{\mathfrak{g}}} \operatorname{ad}_{x}=\operatorname{ad}_{\phi_{\mathfrak{g}}(x)}, \quad\left[D, \operatorname{ad}_{x}\right]_{\phi_{\mathfrak{g}}}=\operatorname{ad}_{D(x)}
$$

Therefore, $\operatorname{Inn}(\mathfrak{g})$ is an ideal of the Hom-Lie algebra $\left(\operatorname{Der}(\mathfrak{g}),[\cdot, \cdot]_{\phi_{\mathfrak{g}}}, \operatorname{Ad}_{\phi_{\mathfrak{g}}}\right)$.

Denote by $\operatorname{Out}(\mathfrak{g})$ the set of out derivations of the Hom-Lie algebra $\left(\mathfrak{g},[\cdot, \cdot]_{\mathfrak{g}}, \phi_{\mathfrak{g}}\right)$, that is,

$$
\operatorname{Out}(\mathfrak{g})=\operatorname{Der}(\mathfrak{g}) / \operatorname{lnn}(\mathfrak{g})
$$

We use $\pi$ to denote the quotient map from $\operatorname{Der}(\mathfrak{g})$ to $\operatorname{Out}(\mathfrak{g})$.

\subsection{Non-Abelian Extensions of Hom-Lie Algebras}

Definition 5. A non-abelian extension of a Hom-Lie algebra $\left(\mathfrak{g},[\cdot, \cdot]_{\mathfrak{g}}, \phi_{\mathfrak{g}}\right)$ by a Hom-Lie algebra $\left(\mathfrak{h},[\cdot, \cdot]_{\mathfrak{h}}, \phi_{\mathfrak{h}}\right)$ is a commutative diagram with rows being short exact sequences of Hom-Lie algebra morphisms:

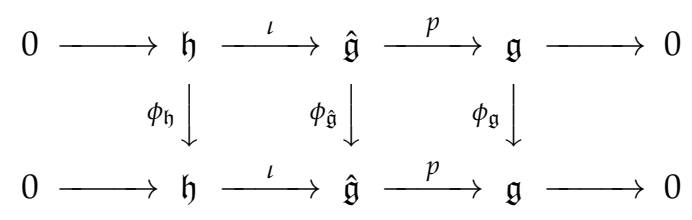

where $\left(\hat{\mathfrak{g}},[\cdot, \cdot] \hat{\mathfrak{g}}, \phi_{\hat{\mathfrak{g}}}\right)$ is a Hom-Lie algebra.

We can regard $\mathfrak{h}$ as a subspace of $\hat{\mathfrak{g}}$ and $\left.\phi_{\hat{\mathfrak{g}}}\right|_{\mathfrak{h}}=\phi_{\mathfrak{h}}$. Thus, $\mathfrak{h}$ is an invariant subspace of $\phi_{\hat{\mathfrak{g}}}$. We say that an extension is diagonal if $\mathfrak{g}$ has an invariant subspace $X$ of $\phi_{\mathfrak{g}}$ such that $\mathfrak{h} \oplus X=\hat{\mathfrak{g}}$. In general, $\hat{\mathfrak{g}}$ does not always have an invariant subspace $X$ of $\phi_{\hat{\mathfrak{g}}}$ such that $\mathfrak{h} \oplus X=\hat{\mathfrak{g}}$. For example, the matrix representation of $\phi_{\hat{\mathfrak{g}}}$ is a Jordan block. We only study diagonal non-abelian extensions in the sequel. 
Definition 6. Two extensions of $\mathfrak{g}$ by $\mathfrak{h},\left(\hat{\mathfrak{g}}_{1},[\cdot, \cdot]_{\hat{\mathfrak{g}}_{1},} \phi_{\hat{\mathfrak{g}}_{1}}\right)$ and $\left(\hat{\mathfrak{g}}_{2},[\cdot, \cdot]_{\hat{\mathfrak{g}}_{2}}, \phi_{\hat{\mathfrak{g}}_{2}}\right)$ are said to be isomorphic if there exists a Hom-Lie algebra morphism $\theta: \hat{\mathfrak{g}}_{2} \rightarrow \hat{\mathfrak{g}}_{1}$ such that we have the following commutative diagram:

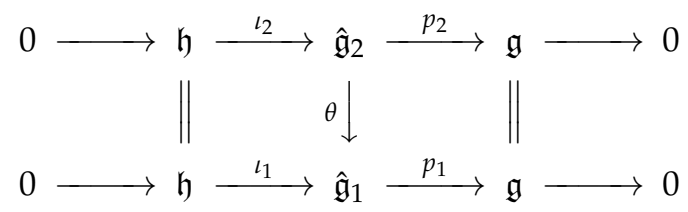

Lemma 2 ([14], (Lemma 4.4)). A Hom-Lie algebra $\left.(\hat{\mathfrak{g}},[\cdot, \cdot]]_{\hat{\mathfrak{g}}}, \phi_{\hat{\mathfrak{g}}}\right)$ is a diagonal non-abelian extension of a Hom-Lie algebra $\left(\mathfrak{g},[\cdot, \cdot]_{\mathfrak{g}}, \phi_{\mathfrak{g}}\right)$ by a Hom-Lie algebra $\left(\mathfrak{h},[\cdot, \cdot]_{\mathfrak{h}}, \phi_{\mathfrak{h}}\right)$ if and only if there is a section $s: \mathfrak{g} \rightarrow \hat{\mathfrak{g}}$ such that $p \circ s=\mathrm{Id}$ and $\phi_{\hat{\mathfrak{g}}} \circ s=s \circ \phi_{\mathfrak{g}}$. This section is called diagonal.

Let $\left(\hat{\mathfrak{g}},[\cdot, \cdot]_{\hat{\mathfrak{g}}}, \phi_{\hat{\mathfrak{g}}}\right)$ be a diagonal extension of a Hom-Lie algebra $\left(\mathfrak{g},[\cdot, \cdot]_{\mathfrak{g}}, \phi_{\mathfrak{g}}\right)$ by a Hom-Lie algebra $\left(\mathfrak{h},[\cdot, \cdot]_{\mathfrak{h}}, \phi_{\mathfrak{h}}\right)$ and $s: \mathfrak{g} \rightarrow \hat{\mathfrak{g}}$ be a diagonal section. Define linear maps $\omega: \mathfrak{g} \wedge \mathfrak{g} \rightarrow \mathfrak{h}$ and $\rho: \mathfrak{g} \rightarrow \mathfrak{g l}(\mathfrak{h})$ respectively by

$$
\begin{aligned}
\omega(x, y) & =[s(x), s(y)]_{\hat{\mathfrak{g}}}-s[x, y]_{\mathfrak{g}}, \quad \forall x, y \in \mathfrak{g} \\
\rho_{x}(u) & =[s(x), u]_{\hat{\mathfrak{g}}}, \quad \forall x \in \mathfrak{g}, u \in \mathfrak{h}
\end{aligned}
$$

Clearly, $\hat{\mathfrak{g}}$ is isomorphic to $\mathfrak{g} \oplus \mathfrak{h}$ as vector spaces. Transferring the Hom-Lie algebra structure on $\hat{\mathfrak{g}}$ to that on $\mathfrak{g} \oplus \mathfrak{h}$, we obtain a Hom-Lie algebra $\left(\mathfrak{g} \oplus \mathfrak{h},[\cdot, \cdot]_{(\rho, \omega)}, \phi\right)$, where $[\cdot, \cdot]_{(\rho, \omega)}$ and $\phi$ are given by

$$
\begin{aligned}
{[x+u, y+v]_{(\rho, \omega)} } & =[x, y]_{\mathfrak{g}}+\omega(x, y)+\rho_{x}(v)-\rho_{y}(u)+[u, v]_{\mathfrak{h}} \\
\phi(x+u) & =\phi_{\mathfrak{g}}(x)+\phi_{\mathfrak{h}}(u)
\end{aligned}
$$

The following proposition gives the conditions on $\rho$ and $\omega$ such that $\left(\mathfrak{g} \oplus \mathfrak{h},[\cdot, \cdot]_{(\rho, \omega)}, \phi\right)$ is a Hom-Lie algebra.

Proposition 1 ([14], (Proposition 4.5)). With the above notations, $\left(\mathfrak{g} \oplus \mathfrak{h},[\cdot, \cdot]_{(\rho, \omega)}, \phi\right)$ is a Hom-Lie algebra if and only if $\rho$ and $\omega$ satisfy the following equalities:

$$
\begin{aligned}
\phi_{\mathfrak{h}} \circ \rho_{x} & =\rho_{\phi_{\mathfrak{g}}(x)} \circ \phi_{\mathfrak{h}} \\
\rho_{x}\left([u, v]_{\mathfrak{h}}\right) & =\left[\phi_{\mathfrak{h}}(u),\left(\operatorname{Ad}_{\phi_{\mathfrak{h}}^{-1}} \rho_{x}\right)(v)\right]_{\mathfrak{h}}+\left[\left(\operatorname{Ad}_{\phi_{\mathfrak{h}}^{-1}} \rho_{x}\right)(u), \phi_{\mathfrak{h}}(v)\right]_{\mathfrak{h}} \\
\phi_{\mathfrak{h}} \circ \omega & =\omega \circ \phi_{\mathfrak{g}}^{\otimes 2} \\
{\left[\rho_{x}, \rho_{y}\right]_{\phi_{\mathfrak{h}}}-\rho_{[x, y]_{\mathfrak{g}}} } & =\operatorname{ad}_{\omega(x, y)} \\
\rho_{\phi_{\mathfrak{g}}(x)}(\omega(y, z))+\text { c.p. } & =\omega\left([x, y]_{\mathfrak{g}}, \phi_{\mathfrak{g}}(z)\right)+\text { c.p. }
\end{aligned}
$$

where c.p. is the cyclic permutation of $x, y, z$.

For any diagonal non-abelian extension, by choosing a diagonal section, it is isomorphic to $\left(\mathfrak{g} \oplus \mathfrak{h},[\cdot, \cdot]_{(\rho, \omega)}, \phi\right)$. Therefore, we only consider diagonal non-abelian extensions of the form $(\mathfrak{g} \oplus$ $\left.\mathfrak{h},[\cdot, \cdot]_{(\rho, \omega)}, \phi\right)$ in the sequel.

Proposition 2 ([14], (Theorem 4.9)). Let $\left(\mathfrak{g} \oplus \mathfrak{h},[\cdot, \cdot]_{(\rho, \omega)}, \phi\right)$ and $\left(\mathfrak{g} \oplus \mathfrak{h},[\cdot, \cdot]_{\left(\rho^{\prime}, \omega^{\prime}\right)}, \phi\right)$ be two diagonal non-abelian extensions of $\mathfrak{g}$ by $\mathfrak{h}$. The two extensions are equivalent if and only if there is a linear map $\xi: \mathfrak{g} \rightarrow \mathfrak{h}$ such that

$$
\begin{aligned}
\phi_{\mathfrak{h}}(\xi(x)) & =\xi\left(\phi_{\mathfrak{g}}(x)\right) \\
\rho_{x}^{\prime}-\rho_{x} & =\operatorname{ad}_{\xi(x)} \\
\omega^{\prime}(x, y)-\omega(x, y) & =\rho_{x}(\xi(y))-\rho_{y}(\xi(x))+[\xi(x), \xi(y)]_{\mathfrak{h}}-\xi\left([x, y]_{\mathfrak{g}}\right)
\end{aligned}
$$




\section{Classification of Diagonal Non-Abelian Extensions of Hom-Lie Algebras: Special Case}

In this section, we classify diagonal non-abelian extensions of Hom-Lie algebras for the case that $\operatorname{Cen}(\mathfrak{h})=0$.

Theorem 2. Let $\left(\mathfrak{g},[\cdot, \cdot]_{\mathfrak{g}}, \phi_{\mathfrak{g}}\right)$ and $\left(\mathfrak{h},[\cdot, \cdot]_{\mathfrak{h}}, \phi_{\mathfrak{h}}\right)$ be Hom-Lie algebras such that $\operatorname{Cen}(\mathfrak{h})=0$. If the following short exact sequence of Hom-Lie algebra morphisms:

$$
0 \rightarrow \operatorname{lnn}(\mathfrak{h}) \rightarrow \operatorname{Der}(\mathfrak{h}) \stackrel{\pi}{\rightarrow} \operatorname{Out}(\mathfrak{h}) \rightarrow 0
$$

is a diagonal non-abelian extension of $\operatorname{Out}(\mathfrak{h})$ by $\operatorname{Inn}(\mathfrak{h})$, then isomorphism classes of diagonal non-abelian extensions of $\mathfrak{g}$ by $\mathfrak{h}$ correspond bijectively to Hom-Lie algebra homomorphisms:

$$
\bar{\rho}: \mathfrak{g} \rightarrow \operatorname{Out}(\mathfrak{h})
$$

Proof. Let $\left(\mathfrak{g} \oplus \mathfrak{h},[\cdot, \cdot]_{(\rho, \omega)}, \phi\right)$ be a diagonal non-abelian extension of $\mathfrak{g}$ by $\mathfrak{h}$ given by Equations (15) and (16). By Equation (18), we have $\rho_{x} \in \operatorname{Der}(\mathfrak{h})$. Let $\pi: \operatorname{Der}(\mathfrak{h}) \rightarrow \operatorname{Out}(\mathfrak{h})$ be the quotient map. We denote the induced Hom-Lie algebra structure on Out $(\mathfrak{h})$ by $[\cdot, \cdot]_{\phi_{\mathfrak{h}}}^{\prime}$ and $\operatorname{Ad}_{\phi_{\mathfrak{h}}}^{\prime}$. Hence we can define

$$
\bar{\rho}=\pi \circ \rho
$$

By Equation (17), for all $x \in \mathfrak{g}$, we have

$$
\bar{\rho}_{\phi_{\mathfrak{g}}(x)}=\pi\left(\rho_{\phi_{\mathfrak{g}}(x)}\right)=\pi\left(\operatorname{Ad}_{\phi_{\mathfrak{h}}}\left(\rho_{x}\right)\right)=\operatorname{Ad}_{\phi_{\mathfrak{h}}}^{\prime}\left(\bar{\rho}_{x}\right)
$$

By Equation (20), we have

$$
\bar{\rho}_{[x, y]_{\mathfrak{g}}}=\pi\left(\left[\rho_{x}, \rho_{y}\right]_{\phi_{\mathfrak{h}}}-\operatorname{ad}_{\omega(x, y)}\right)=\pi\left(\left[\rho_{x}, \rho_{y}\right]_{\phi_{\mathfrak{h}}}\right)=\left[\bar{\rho}_{x}, \bar{\rho}_{y}\right]_{\phi_{\mathfrak{h}}}^{\prime}
$$

Thus, $\bar{\rho}$ is a Hom-Lie algebra homomorphism from $\mathfrak{g}$ to $\operatorname{Out}(\mathfrak{h})$.

Let $\left(\mathfrak{g} \oplus \mathfrak{h},[\cdot, \cdot]_{\left(\rho^{\prime}, \omega^{\prime}\right)}, \phi\right)$ and $\left(\mathfrak{g} \oplus \mathfrak{h},[\cdot, \cdot]_{(\rho, \omega)}, \phi\right)$ be isomorphic diagonal non-abelian extensions of $\mathfrak{g}$ by $\mathfrak{h}$. By Proposition 2, we have

$$
\bar{\rho}_{x}^{\prime}=\pi\left(\rho_{x}+\operatorname{ad}_{\tilde{\xi}(x)}\right)=\bar{\rho}_{x}
$$

Thus, we obtain that isomorphic diagonal non-abelian extensions of $\mathfrak{g}$ by $\mathfrak{h}$ correspond to the same Hom-Lie algebra homomorphism from $\mathfrak{g}$ to Out $(\mathfrak{h})$.

Conversely, let $\bar{\rho}$ be a Hom-Lie algebra homomorphism from $\mathfrak{g}$ to Out $(\mathfrak{h})$. Because the short exact sequence of Hom-Lie algebras (Equation (25)) is a diagonal non-abelian extension of $\operatorname{Out}(\mathfrak{h})$ by $\operatorname{lnn}(\mathfrak{h})$, we can choose a diagonal section $s$ of $\pi: \operatorname{Der}(\mathfrak{g}) \rightarrow \operatorname{Out}(\mathfrak{h})$. Moreover, we define $\rho: \mathfrak{g} \rightarrow \mathfrak{g l}(\mathfrak{h})$ by

$$
\rho_{x}=s\left(\bar{\rho}_{x}\right)
$$

We have $\rho_{x} \in \operatorname{Der}(\mathfrak{h})$. Thus we obtain Equation (18). Because $s$ is a diagonal section, we have

$$
\begin{aligned}
\rho_{\phi_{\mathfrak{g}}(x)} \circ \phi_{\mathfrak{h}} & \left.=\left(s\left(\bar{\rho}_{\phi_{\mathfrak{g}}(x)}\right)\right) \circ \phi_{\mathfrak{h}}=\left(s\left(\operatorname{Ad}_{\phi_{\mathfrak{h}}}^{\prime}\left(\bar{\rho}_{x}\right)\right)\right) \circ \phi_{\mathfrak{h}}=\left(\left(s \circ \operatorname{Ad}_{\phi_{\mathfrak{h}}}^{\prime}\right) \circ \bar{\rho}_{x}\right)\right) \circ \phi_{\mathfrak{h}} \\
& \left.=\left(\left(\operatorname{Ad}_{\phi_{\mathfrak{h}}} \circ s\right) \circ \bar{\rho}_{x}\right)\right) \circ \phi_{\mathfrak{h}}=\left(\operatorname{Ad}_{\phi_{\mathfrak{h}}}\left(s\left(\bar{\rho}_{x}\right)\right)\right) \circ \phi_{\mathfrak{h}}=\phi_{\mathfrak{h}} \circ s\left(\bar{\rho}_{x}\right) \circ \phi_{\mathfrak{h}}^{-1} \circ \phi_{\mathfrak{h}} \\
& =\phi_{\mathfrak{h}} \circ \rho_{x}
\end{aligned}
$$

Thus, we obtain Equation (17). Because $\pi$ and $\bar{\rho}$ are Hom-Lie algebra homomorphisms, for all $x, y \in \mathfrak{g}$, we have

$$
\pi\left(\left[\rho_{x}, \rho_{y}\right]_{\phi_{\mathfrak{h}}}-\rho_{[x, y]_{\mathfrak{g}}}\right)=\left[\pi\left(\rho_{x}\right), \pi\left(\rho_{y}\right)\right]_{\phi_{\mathfrak{h}}}^{\prime}-\pi\left(\rho_{[x, y]_{\mathfrak{g}}}\right)=\left[\bar{\rho}_{x}, \bar{\rho}_{y}\right]_{\phi_{\mathfrak{h}}}^{\prime}-\bar{\rho}_{[x, y]_{\mathfrak{g}}}=0
$$


which implies that $\left[\rho_{x}, \rho_{y}\right]_{\phi_{\mathfrak{h}}}-\rho_{[x, y]_{\mathfrak{g}}} \in \operatorname{Inn}(\mathfrak{h})$. Because we have the following short exact sequence of Hom-Lie algebra morphisms:

$$
0 \rightarrow \operatorname{Cen}(\mathfrak{h}) \rightarrow \mathfrak{h} \stackrel{\text { ad }}{\rightarrow} \operatorname{Inn}(\mathfrak{h}) \rightarrow 0
$$

and $\operatorname{Cen}(\mathfrak{h})=0$, there exists a unique linear map $\omega: \mathfrak{g} \wedge \mathfrak{g} \rightarrow \mathfrak{h}$ such that

$$
\left[\rho_{x}, \rho_{y}\right]_{\phi_{\mathfrak{h}}}-\rho_{[x, y]_{\mathfrak{g}}}=\operatorname{ad}_{\omega(x, y)}
$$

Furthermore, we claim that

$$
\phi_{\mathfrak{h}} \circ \omega=\omega \circ \phi_{\mathfrak{g}}^{\otimes 2}
$$

In fact, for all $u \in \mathfrak{h}$, we have

$$
\begin{aligned}
& {\left[\phi_{\mathfrak{h}}(\omega(x, y))-\omega\left(\phi_{\mathfrak{g}}(x), \phi_{\mathfrak{g}}(y)\right), \phi_{\mathfrak{h}}(u)\right]_{\mathfrak{h}} } \\
= & \phi_{\mathfrak{h}} \operatorname{ad}_{\omega(x, y)} u-\operatorname{ad}_{\omega\left(\phi_{\mathfrak{g}}(x), \phi_{\mathfrak{g}}(y)\right)} \phi_{\mathfrak{h}}(u) \\
= & \phi_{\mathfrak{h}}\left(\left[\rho_{x}, \rho_{y}\right]_{\phi_{\mathfrak{h}}}(u)-\rho_{[x, y]_{\mathfrak{g}}}(u)\right)-\left(\left[\rho_{\phi_{\mathfrak{g}}(x)}, \rho_{\phi_{\mathfrak{g}}(y)}\right]_{\phi_{\mathfrak{h}}}\left(\phi_{\mathfrak{h}}(u)\right)-\rho_{\phi_{\mathfrak{g}}[x, y]_{\mathfrak{g}}}\left(\phi_{\mathfrak{h}}(u)\right)\right) \\
= & 0
\end{aligned}
$$

which implies that $\phi_{\mathfrak{h}}(\omega(x, y))-\omega\left(\phi_{\mathfrak{g}}(x), \phi_{\mathfrak{g}}(y)\right)=0$ because Cen $(\mathfrak{h})=0$. Thus, we obtain Equations (19) and (20). For all $x, y, z \in \mathfrak{g}, u \in \mathfrak{h}$, by $\rho_{x} \in \operatorname{Der}(\mathfrak{h})$ and Equations (27) and (28), we have

$$
\begin{aligned}
& {\left[\left(\rho_{\phi_{\mathfrak{g}}(x)}(\omega(y, z))-\omega\left([x, y]_{\mathfrak{g}}, \phi_{\mathfrak{g}}(z)\right)\right)+c . p ., \phi_{\mathfrak{h}}(u)\right]_{\mathfrak{h}}} \\
& =\phi_{\mathfrak{h}}\left(\left[\left(\operatorname{Ad}_{\phi_{\mathfrak{h}}^{-1}} \rho_{\phi_{\mathfrak{g}}(x)}\right)\left(\omega\left(\phi_{\mathfrak{g}}^{-1} y, \phi_{\mathfrak{g}}^{-1} z\right)\right), \phi_{\mathfrak{h}}\left(\phi_{\mathfrak{h}}^{-1} u\right)\right]_{\mathfrak{h}}-\left[\omega\left(\left[\phi_{\mathfrak{g}}^{-1} x, \phi_{\mathfrak{g}}^{-1} y\right]_{\mathfrak{g}}, z\right), u\right]_{\mathfrak{h}}+c . p .\right) \\
& =\phi_{\mathfrak{h}}\left(\rho_{\phi_{\mathfrak{g}}(x)}\left[\omega\left(\phi_{\mathfrak{g}}^{-1} y, \phi_{\mathfrak{g}}^{-1} z\right), \phi_{\mathfrak{h}}^{-1} u\right]_{\mathfrak{h}}-\left[\omega(y, z),\left(\operatorname{Ad}_{\phi_{\mathfrak{h}}^{-1}} \rho_{\phi_{\mathfrak{g}}(x)}\right)\left(\phi_{\mathfrak{h}}^{-1} u\right)\right]_{\mathfrak{h}}\right. \\
& \left.-\left[\rho_{\left[\phi_{\mathfrak{g}}^{-1} x, \phi_{\mathfrak{g}}^{-1} y\right]_{\mathfrak{g}}} \rho_{z}\right]_{\phi_{\mathfrak{h}}}(u)+\rho_{\left[\left[\phi_{\mathfrak{g}}^{-1} x, \phi_{\mathfrak{g}}^{-1} y\right]_{\mathfrak{g}}, z\right]_{\mathfrak{g}}}(u)+c . p .\right) \\
& =\phi_{\mathfrak{h}}\left(\rho_{\phi_{\mathfrak{g}}(x)}\left(\left(\left[\rho_{\phi_{\mathfrak{g}}^{-1} y^{\prime}} \rho_{\phi_{\mathfrak{g}}^{-1} z}\right]_{\phi_{\mathfrak{h}}}-\rho_{\left[\phi_{\mathfrak{g}}^{-1} y, \phi_{\mathfrak{g}}^{-1} z\right]_{\mathfrak{g}}}\right)\left(\phi_{\mathfrak{h}}^{-1} u\right)\right)-\left(\left[\rho_{y}, \rho_{z}\right]_{\phi_{\mathfrak{h}}}-\rho_{[y, z]_{\mathfrak{g}}}\right)\left(\left(\rho_{x} \circ \phi_{\mathfrak{h}}^{-1}\right)(u)\right)\right. \\
& \left.-\left[\rho_{\left[\phi_{\mathfrak{g}}^{-1} x, \phi_{\mathfrak{g}}^{-1} y\right]_{\mathfrak{g}}} \rho_{z}\right]_{\phi_{\mathfrak{h}}}(u)+\rho_{\left[\left[\phi_{\mathfrak{g}}^{-1} x, \phi_{\mathfrak{g}}^{-1} y\right]_{\mathfrak{g}}, z\right]_{\mathfrak{g}}}(u)+c . p .\right) \\
& =\phi_{\mathfrak{h}}\left(\left(\phi_{\mathfrak{h}} \circ \rho_{x} \circ \phi_{\mathfrak{h}}^{-1} \circ\left[\rho_{\phi_{\mathfrak{g}}^{-1} y^{\prime}} \rho_{\phi_{\mathfrak{g}}^{-1} z}\right]_{\phi_{\mathfrak{h}}} \circ \phi_{\mathfrak{h}}^{-1}-\phi_{\mathfrak{h}} \circ\left[\rho_{\phi_{\mathfrak{g}}^{-1} y^{\prime}} \rho_{\phi_{\mathfrak{g}}^{-1} z}\right]_{\phi_{\mathfrak{h}}} \circ \phi_{\mathfrak{h}}^{-1} \circ \rho_{x} \circ \phi_{\mathfrak{h}}^{-1}\right)(u)\right. \\
& +\left(\phi_{\mathfrak{h}} \circ \rho_{\left[\phi_{\mathfrak{g}}^{-1} y, \phi_{\mathfrak{g}}^{-1} z\right]_{\mathfrak{g}}} \circ \phi_{\mathfrak{h}}^{-1} \circ \rho_{x} \circ \phi_{\mathfrak{h}}^{-1}-\phi_{\mathfrak{h}} \circ \rho_{x} \circ \phi_{\mathfrak{h}}^{-1} \circ \rho_{\left[\phi_{\mathfrak{g}}^{-1} y, \phi_{\mathfrak{g}}^{-1} z\right]_{\mathfrak{g}}} \circ \phi_{\mathfrak{h}}^{-1}\right)(u) \\
& \left.-\left[\rho_{\left[\phi_{\mathfrak{g}}^{-1} x, \phi_{\mathfrak{g}}^{-1} y\right]_{\mathfrak{g}}} \rho_{z}\right]_{\phi_{\mathfrak{h}}}(u)+\rho_{\left[\left[\phi_{\mathfrak{g}}^{-1} x, \phi_{\mathfrak{g}}^{-1} y\right]_{\mathfrak{g}}, z\right]_{\mathfrak{g}}}(u)+c . p .\right) \\
& =\phi_{\mathfrak{h}}\left(\left(\left[\rho_{x},\left[\rho_{\phi_{\mathfrak{g}}^{-1} y^{\prime}} \rho_{\phi_{\mathfrak{g}}^{-1} z}\right]_{\phi_{\mathfrak{h}}}\right]_{\phi_{\mathfrak{h}}}+\left[\rho_{\left[\phi_{\mathfrak{g}}^{-1} y, \phi_{\mathfrak{g}}^{-1} z\right]_{\mathfrak{g}}} \rho_{x}\right]_{\phi_{\mathfrak{h}}}\right.\right. \\
& \left.\left.-\left[\rho_{\left[\phi_{\mathfrak{g}}^{-1} x, \phi_{\mathfrak{g}}^{-1} y\right]_{\mathfrak{g}}}, \rho_{z}\right]_{\phi_{\mathfrak{h}}}+\rho_{\left[\left[\phi_{\mathfrak{g}}^{-1} x, \phi_{\mathfrak{g}}^{-1} y\right]_{\mathfrak{g}} z\right]_{\mathfrak{g}}}\right)(u)+\text { c.p. }\right) \\
& =0
\end{aligned}
$$

Thus, we have

$$
\rho_{\phi_{\mathfrak{g}}(x)}(\omega(y, z))-\omega\left([x, y]_{\mathfrak{g}}, \phi_{\mathfrak{g}}(z)\right)+\text { c.p. } \in \operatorname{Cen}(\mathfrak{h})
$$

Because Cen $(\mathfrak{h})=0$, we have Equation (21). Therefore, we deduce that Equations (17)-(21) hold. By Proposition $1,\left(\mathfrak{g} \oplus \mathfrak{h},[\cdot, \cdot]_{(\rho, \omega)}, \phi\right)$ is a diagonal non-abelian extension of $\mathfrak{g}$ by $\mathfrak{h}$.

If we choose another section $s^{\prime}$ of $\pi: \operatorname{Der}(\mathfrak{g}) \rightarrow \operatorname{Out}(\mathfrak{h})$, we obtain another diagonal non-abelian extension $\left(\mathfrak{g} \oplus \mathfrak{h},[\cdot, \cdot]_{\left(\rho^{\prime}, \omega^{\prime}\right)}, \phi\right)$. Clearly, we have

$$
\pi\left(\rho_{x}^{\prime}-\rho_{x}\right)=\left(\pi \circ s^{\prime}\right)\left(\bar{\rho}_{x}\right)-(\pi \circ s)\left(\bar{\rho}_{x}\right)=0
$$


which implies that $\rho_{x}^{\prime}-\rho_{x} \in \operatorname{lnn}(\mathfrak{h})$. Because $\operatorname{Cen}(\mathfrak{h})=0$, there is a unique linear map $\xi: \mathfrak{g} \rightarrow \mathfrak{h}$ such that

$$
\rho_{x}^{\prime}-\rho_{x}=\operatorname{ad}_{\tilde{\zeta}(x)}
$$

We claim that

$$
\phi_{\mathfrak{h}}(\xi(x))=\xi\left(\phi_{\mathfrak{g}}(x)\right)
$$

In fact, for all $u \in \mathfrak{h}$, we have

$$
\begin{aligned}
{\left[\phi_{\mathfrak{h}}(\xi(x))-\xi\left(\phi_{\mathfrak{g}}(x)\right), \phi_{\mathfrak{h}}(u)\right]_{\mathfrak{h}} } & =\phi_{\mathfrak{h}} \operatorname{ad}_{\tilde{\xi}(x)} u-\operatorname{ad}_{\tilde{\zeta}\left(\phi_{\mathfrak{g}}(x)\right)} \phi_{\mathfrak{h}}(u) \\
& =\phi_{\mathfrak{h}}\left(\rho_{x}^{\prime} u-\rho_{x} u\right)-\left(\rho_{\phi_{\mathfrak{g}}(x)}^{\prime} \phi_{\mathfrak{h}}(u)-\rho_{\phi_{\mathfrak{g}}(x)} \phi_{\mathfrak{h}}(u)\right) \\
& =0
\end{aligned}
$$

which implies that $\phi_{\mathfrak{h}}(\xi(x))=\xi\left(\phi_{\mathfrak{g}}(x)\right)$ because $\operatorname{Cen}(\mathfrak{h})=0$. Thus, we obtain Equations (22) and (23). By Lemma 1 and Equation (28), for all $x, y \in \mathfrak{g}$ we have

$$
\begin{aligned}
\operatorname{ad}_{\omega^{\prime}(x, y)-\omega(x, y)} & =\left[\rho_{x}^{\prime}, \rho_{y}^{\prime}\right]_{\phi_{\mathfrak{h}}}-\rho_{[x, y]_{\mathfrak{g}}}^{\prime}-\left[\rho_{x}, \rho_{y}\right]_{\phi_{\mathfrak{h}}}+\rho_{[x, y]_{\mathfrak{g}}} \\
& =\left[\rho_{x}+\operatorname{ad}_{\tilde{\xi}(x)}, \rho_{y}+\operatorname{ad}_{\tilde{\xi}(y)}\right]_{\phi_{\mathfrak{h}}}-\rho_{[x, y]_{\mathfrak{g}}}-\operatorname{ad}_{\tilde{\xi}\left([x, y]_{\mathfrak{g}}\right)}-\left[\rho_{x}, \rho_{y}\right]_{\phi_{\mathfrak{h}}}+\rho_{[x, y]_{\mathfrak{g}}} \\
& =\left[\rho_{x}, \operatorname{ad}_{\tilde{\xi}(y)}\right]_{\phi_{\mathfrak{h}}}+\left[\operatorname{ad}_{\tilde{\xi}(x)}, \rho_{y}\right]_{\phi_{\mathfrak{h}}}+\left[\operatorname{ad}_{\tilde{\xi}(x)}, \operatorname{ad}_{\tilde{\xi}(y)}\right]_{\phi_{\mathfrak{h}}}-\operatorname{ad}_{\xi\left([x, y]_{\mathfrak{g}}\right)} \\
& =\operatorname{ad}_{\rho_{x}(\tilde{\xi}(y))}-\operatorname{ad}_{\rho_{y}(\xi(x))}+\operatorname{ad}_{[\xi(x), \xi(y)]_{\mathfrak{h}}}-\operatorname{ad}_{\tilde{\xi}\left([x, y]_{\mathfrak{g}}\right)} \\
& =\operatorname{ad}_{\rho_{x}(\xi(y))-\rho_{y}(\tilde{\xi}(x))+[\xi(x), \xi(y)]_{\mathfrak{h}}-\xi\left([x, y]_{\mathfrak{g}}\right)}
\end{aligned}
$$

By $\operatorname{Cen}(\mathfrak{h})=0$, we obtain Equation (24). Thus, we have Equations (22)-(24). Therefore, we deduce that $\left(\mathfrak{g} \oplus \mathfrak{h},[\cdot, \cdot]_{(\rho, \omega)}, \phi\right)$ and $\left(\mathfrak{g} \oplus \mathfrak{h},[\cdot, \cdot]_{\left(\rho^{\prime}, \omega^{\prime}\right)}, \phi\right)$ are isomorphic diagonal non-abelian extensions of $\mathfrak{g}$ by $\mathfrak{h}$. The proof is finished.

\section{Obstruction of Existence of Diagonal Non-Abelian Extensions of Hom-Lie Algebras}

In this section, we always assume that the following short exact sequences of Hom-Lie algebra morphisms:

$$
\begin{array}{r}
0 \rightarrow \operatorname{Inn}(\mathfrak{h}) \rightarrow \operatorname{Der}(\mathfrak{h}) \stackrel{\pi}{\rightarrow} \operatorname{Out}(\mathfrak{h}) \rightarrow 0 \\
0 \rightarrow \operatorname{Cen}(\mathfrak{h}) \rightarrow \mathfrak{h} \stackrel{\text { ad }}{\rightarrow} \operatorname{Inn}(\mathfrak{h}) \rightarrow 0
\end{array}
$$

are diagonal non-abelian extensions. Given a Hom-Lie algebra morphism $\bar{\rho}: \mathfrak{g} \rightarrow$ Out(h), where $\operatorname{Cen}(\mathfrak{h}) \neq 0$, we consider the obstruction of existence of non-abelian extensions. By choosing a diagonal section $s$ of $\pi: \operatorname{Der}(\mathfrak{h}) \rightarrow \operatorname{Out}(\mathfrak{h})$, we can still define $\rho$ by Equation (26) such that Equation (27) holds. Moreover, we can choose a linear map $\omega: \mathfrak{g} \wedge \mathfrak{g} \rightarrow \mathfrak{h}$ such that Equations (28) and (29) hold. Thus, $\left(\mathfrak{g} \oplus \mathfrak{h},[\cdot, \cdot]_{(\rho, \omega)}, \phi\right)$ is a diagonal non-abelian extension of $\mathfrak{g}$ by $\mathfrak{h}$ if and only if

$$
\rho_{\phi_{\mathfrak{g}}(x)}(\omega(y, z))-\omega\left([x, y]_{\mathfrak{g}}, \phi_{\mathfrak{g}}(z)\right)+\text { c.p. }(x, y, z)=0
$$

Let $\mathrm{d}_{\rho}$ be the formal coboundary operator associated to $\rho$. Then we have

$$
\left(\mathrm{d}_{\rho} \omega\right)(x, y, z)=\rho_{\phi_{\mathfrak{g}}(x)}(\omega(y, z))-\omega\left([x, y]_{\mathfrak{g}}, \phi_{\mathfrak{g}}(z)\right)+c . p \cdot(x, y, z)
$$

Therefore, $\left(\mathfrak{g} \oplus \mathfrak{h},[\cdot, \cdot]_{(\rho, \omega)}, \phi\right)$ is a diagonal non-abelian extension of $\mathfrak{g}$ by $\mathfrak{h}$ if and only if $\mathrm{d}_{\rho} \omega=0$.

Definition 7. Let $\bar{\rho}: \mathfrak{g} \rightarrow \operatorname{Out}(\mathfrak{h})$ be a Hom-Lie algebra morphism. We call $\bar{\rho}$ an extensible homomorphism if there exists a diagonal section s of $\pi: \operatorname{Der}(\mathfrak{h}) \rightarrow \operatorname{Out}(\mathfrak{h})$ and linear map $\omega: \mathfrak{g} \wedge \mathfrak{g} \rightarrow \mathfrak{h}$ such that Equations (27), (28) and (33) hold. 
For all $u \in \operatorname{Cen}(\mathfrak{h})$, it is clear that $\phi_{\mathfrak{h}}(u) \in \operatorname{Cen}(\mathfrak{h})$. For $v \in \mathfrak{h}$, we have

$$
\begin{aligned}
{\left[\rho_{x}(u), v\right]_{\mathfrak{h}} } & =\left[\left(\operatorname{Ad}_{\phi_{\mathfrak{h}}^{-1}} \rho_{\phi_{\mathfrak{g}}(x)}\right)(u), \phi_{\mathfrak{h}}\left(\phi_{\mathfrak{h}}^{-1}(v)\right)\right]_{\mathfrak{h}} \\
& =\rho_{\phi_{\mathfrak{g}}(x)}\left(\left[u, \phi_{\mathfrak{h}}^{-1}(v)\right]_{\mathfrak{h}}\right)-\left[\phi_{\mathfrak{h}}(u),\left(\operatorname{Ad}_{\phi_{\mathfrak{h}}^{-1}} \rho_{\phi_{\mathfrak{g}}(x)}\right)\left(\phi_{\mathfrak{h}}^{-1}(v)\right)\right]_{\mathfrak{h}} \\
& =0
\end{aligned}
$$

Thus, we have $\rho_{x}(u) \in \operatorname{Cen}(\mathfrak{h})$. Therefore, we can define $\hat{\rho}: \mathfrak{g} \rightarrow \mathfrak{g l}(\operatorname{Cen}(\mathfrak{h}))$ by

$$
\left.\hat{\rho}_{x} \triangleq \rho_{x}\right|_{\operatorname{Cen}(\mathfrak{h})}
$$

By Equations (27) and (28), we obtain that $\hat{\rho}$ is a Hom-Lie morphism from $\mathfrak{g}$ to $\mathfrak{g l}(\operatorname{Cen}(\mathfrak{h}))$. By Theorem 1, $\hat{\rho}$ is a representation of $\left(\mathfrak{g},[\cdot, \cdot]_{\mathfrak{g}}, \phi_{\mathfrak{g}}\right)$ on $\operatorname{Cen}(\mathfrak{h})$ with respect to $\left.\phi_{\mathfrak{h}}\right|_{\operatorname{Cen}(\mathfrak{h})}$. By Equation (31), we deduce that different diagonal sections of $\pi$ give the same representation of $\mathfrak{g}$ on $\operatorname{Cen}(\mathfrak{h})$ with respect to $\left.\phi_{\mathfrak{h}}\right|_{\operatorname{Cen}(\mathfrak{h})}$. In the sequel, we always assume that $\hat{\rho}$ is a representation of $\mathfrak{g}$ on $\operatorname{Cen}(\mathfrak{h})$ with respect to $\left.\phi_{\mathfrak{h}}\right|_{\operatorname{Cen}(\mathfrak{h})}$, which is induced by $\bar{\rho}$. By Equation (30), we have

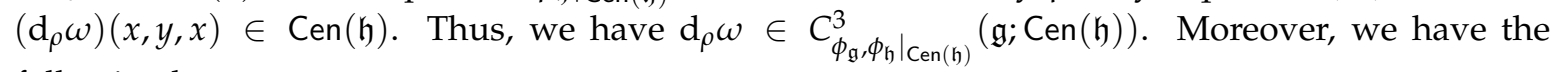
following lemma.

Lemma 3. $\mathrm{d}_{\rho} \omega$ is a 3-cocycle on $\mathfrak{g}$ with the coefficient in $\operatorname{Cen}(\mathfrak{h})$, and the cohomology class $\left[\mathrm{d}_{\rho} \omega\right]$ does not depend on the choices of the diagonal section $s$ of $\pi: \operatorname{Der}(\mathfrak{h}) \rightarrow \operatorname{Out}(\mathfrak{h})$ and $\omega$ that we make.

Proof. For all $x, y, z, t \in \mathfrak{g}$, by straightforward computations, we have

$$
\begin{aligned}
\mathrm{d}_{\hat{\rho}}\left(\mathrm{d}_{\rho} \omega\right)(x, y, z, t)= & \rho_{\phi_{\mathfrak{g}}^{2}(x)}\left(\mathrm{d}_{\rho} \omega(y, z, t)\right)-\rho_{\phi_{\mathfrak{g}}^{2}(y)}\left(\mathrm{d}_{\rho} \omega(x, z, t)\right)+\rho_{\phi_{\mathfrak{g}}^{2}(z)}\left(\mathrm{d}_{\rho} \omega(x, y, t)\right)-\rho_{\phi_{\mathfrak{g}}^{2}(t)}\left(\mathrm{d}_{\rho} \omega(x, y, z)\right) \\
& -\left(\mathrm{d}_{\rho} \omega\right)\left([x, y]_{\mathfrak{g}}, \phi_{\mathfrak{g}}(z), \phi_{\mathfrak{g}}(t)\right)+\left(\mathrm{d}_{\rho} \omega\right)\left([x, z]_{\mathfrak{g}}, \phi_{\mathfrak{g}}(y), \phi_{\mathfrak{g}}(t)\right)-\left(\mathrm{d}_{\rho} \omega\right)\left([x, t]_{\mathfrak{g}}, \phi_{\mathfrak{g}}(y), \phi_{\mathfrak{g}}(z)\right) \\
& -\left(\mathrm{d}_{\rho} \omega\right)\left([y, z]_{\mathfrak{g}}, \phi_{\mathfrak{g}}(x), \phi_{\mathfrak{g}}(t)\right)+\left(\mathrm{d}_{\rho} \omega\right)\left([y, t]_{\mathfrak{g}}, \phi_{\mathfrak{g}}(x), \phi_{\mathfrak{g}}(z)\right)-\left(\mathrm{d}_{\rho} \omega\right)\left([z, t]_{\mathfrak{g}}, \phi_{\mathfrak{g}}(x), \phi_{\mathfrak{g}}(y)\right)
\end{aligned}
$$

By the definition of $\mathrm{d}_{\rho} \omega$, we have 60 terms in the right-hand side of the above formula. Fortunately, we can cancel the following terms:

$$
\begin{aligned}
-\rho_{\phi_{\mathfrak{g}}^{2}(x)}\left(\omega\left([y, z]_{\mathfrak{g}}, \phi_{\mathfrak{g}}(t)\right)\right)-\rho_{\phi_{\mathfrak{g}}^{2}(x)}\left(\omega\left(\phi_{\mathfrak{g}}(t),[y, z]_{\mathfrak{g}}\right)\right) & =0 \\
\omega\left(\left[\phi_{\mathfrak{g}}(z), \phi_{\mathfrak{g}}(t)\right]_{\mathfrak{g}}, \phi_{\mathfrak{g}}\left([x, y]_{\mathfrak{g}}\right)\right)+\omega\left(\left[\phi_{\mathfrak{g}}(x), \phi_{\mathfrak{g}}(y)\right]_{\mathfrak{g}}, \phi_{\mathfrak{g}}\left([z, t]_{\mathfrak{g}}\right)\right) & =0 \\
\omega\left(\left[\phi_{\mathfrak{g}}(t),[x, y]_{\mathfrak{g}}\right]_{\mathfrak{g}}, \phi_{\mathfrak{g}}^{2}(z)\right)+\omega\left(\left[[x, t]_{\mathfrak{g}}, \phi_{\mathfrak{g}}(y)\right]_{\mathfrak{g}}, \phi_{\mathfrak{g}}^{2}(z)\right)-\omega\left(\left[[y, t]_{\mathfrak{g}}, \phi_{\mathfrak{g}}(x)\right]_{\mathfrak{g}}, \phi_{\mathfrak{g}}^{2}(z)\right) & =0
\end{aligned}
$$

By Equations (27)-(29), the above formula reduces to the following:

$$
\begin{aligned}
& \left(\left[\rho_{\phi_{\mathfrak{g}}(x)}, \rho_{\phi_{\mathfrak{g}}(y)}\right]_{\phi_{\mathfrak{h}}}-\rho_{\phi_{\mathfrak{g}}\left([x, y]_{\mathfrak{g}}\right)}\right)\left(\omega\left(\phi_{\mathfrak{g}}(z), \phi_{\mathfrak{g}}(t)\right)\right)+\left(\left[\rho_{\phi_{\mathfrak{g}}(x)}, \rho_{\phi_{\mathfrak{g}}(z)}\right]_{\phi_{\mathfrak{h}}}-\rho_{\phi_{\mathfrak{g}}\left([x, z]_{\mathfrak{g}}\right.}\right)\left(\omega\left(\phi_{\mathfrak{g}}(t), \phi_{\mathfrak{g}}(y)\right)\right) \\
& +\left(\left[\rho_{\phi_{\mathfrak{g}}(x)}, \rho_{\phi_{\mathfrak{g}}(t)}\right]_{\phi_{\mathfrak{h}}}-\rho_{\phi_{\mathfrak{g}}\left([x,]_{\mathfrak{g}}\right)}\right)\left(\omega\left(\phi_{\mathfrak{g}}(y), \phi_{\mathfrak{g}}(z)\right)\right)+\left(\left[\rho_{\phi_{\mathfrak{g}}(y)}, \rho_{\phi_{\mathfrak{g}}(z)}\right]_{\phi_{\mathfrak{h}}}-\rho_{\phi_{\mathfrak{g}}}\left([y, z]_{\mathfrak{g}}\right)\left(\omega\left(\phi_{\mathfrak{g}}(x), \phi_{\mathfrak{g}}(t)\right)\right)\right. \\
& -\left(\left[\rho_{\phi_{\mathfrak{g}}(y)}, \rho_{\phi_{\mathfrak{g}}(t)}\right]_{\phi_{\mathfrak{h}}}+\rho_{\phi_{\mathfrak{g}}\left([y, t]_{\mathfrak{g}}\right)}\right)\left(\omega\left(\phi_{\mathfrak{g}}(x), \phi_{\mathfrak{g}}(z)\right)\right)+\left(\left[\rho_{\phi_{\mathfrak{g}}(z)}, \rho_{\phi_{\mathfrak{g}}(t)}\right]_{\phi_{\mathfrak{h}}}-\rho_{\phi_{\mathfrak{g}}}\left([z, t]_{\mathfrak{g}}\right)\left(\omega\left(\phi_{\mathfrak{g}}(x), \phi_{\mathfrak{g}}(y)\right)\right)\right.
\end{aligned}
$$

Because $\left[\rho_{x}, \rho_{y}\right]_{\phi_{\mathfrak{h}}}-\rho_{[x, y]_{\mathfrak{g}}}=\operatorname{ad}_{\omega(x, y)}$ and $\phi_{\mathfrak{g}}$ is an algebra morphism, this is rewritten as follows:

$$
\begin{aligned}
& {\left[\omega\left(\phi_{\mathfrak{g}}(x), \phi_{\mathfrak{g}}(y)\right), \omega\left(\phi_{\mathfrak{g}}(z), \phi_{\mathfrak{g}}(t)\right)\right]_{\mathfrak{h}}+\left[\omega\left(\phi_{\mathfrak{g}}(x), \phi_{\mathfrak{g}}(z)\right), \omega\left(\phi_{\mathfrak{g}}(t), \phi_{\mathfrak{g}}(y)\right)\right]_{\mathfrak{h}}} \\
& +\left[\omega\left(\phi_{\mathfrak{g}}(x), \phi_{\mathfrak{g}}(t)\right), \omega\left(\phi_{\mathfrak{g}}(y), \phi_{\mathfrak{g}}(z)\right)\right]_{\mathfrak{h}}+\left[\omega\left(\phi_{\mathfrak{g}}(y), \phi_{\mathfrak{g}}(z)\right), \omega\left(\phi_{\mathfrak{g}}(x), \phi_{\mathfrak{g}}(t)\right)\right]_{\mathfrak{h}} \\
& -\left[\omega\left(\phi_{\mathfrak{g}}(y), \phi_{\mathfrak{g}}(t)\right), \omega\left(\phi_{\mathfrak{g}}(x), \phi_{\mathfrak{g}}(z)\right)\right]_{\mathfrak{h}}+\left[\omega\left(\phi_{\mathfrak{g}}(z), \phi_{\mathfrak{g}}(t)\right), \omega\left(\phi_{\mathfrak{g}}(x), \phi_{\mathfrak{g}}(y)\right)\right]_{\mathfrak{h}} \\
& =0
\end{aligned}
$$

Thus, we obtain $\mathrm{d}_{\rho} \omega \in \mathcal{Z}^{3}(\mathfrak{g} ; \hat{\rho})$.

Now we check that the cohomology class $\left[\mathrm{d}_{\rho} \omega\right]$ does not depend on the choices of the diagonal section $s$ of $\pi: \operatorname{Der}(\mathfrak{h}) \rightarrow \operatorname{Out}(\mathfrak{h})$ and $\omega$ that we make. Let $s^{\prime}$ be another diagonal section of $\pi$; we have $\rho_{x}^{\prime} \in \operatorname{Der}(\mathfrak{h})$ and 
choose $\omega^{\prime}$ such that Equations (27) and (28) hold. We prove that $\left[\mathrm{d}_{\rho} \omega\right]=\left[\mathrm{d}_{\rho^{\prime}} \omega^{\prime}\right]$. Because $s$ and $s^{\prime}$ are diagonal sections of $\pi$, we have a linear map $b: \mathfrak{g} \rightarrow \mathfrak{h}$ such that

$$
b \circ \phi_{\mathfrak{g}}=\phi_{\mathfrak{h}} \circ b, \quad \rho_{x}^{\prime}=\rho_{x}+\operatorname{ad}_{b(x)}
$$

We define $\omega^{*}$ by

$$
\omega^{*}(x, y)=\omega(x, y)+\rho_{x}(b(y))-\rho_{y}(b(x))+[b(x), b(y)]_{\mathfrak{h}}-b[x, y]_{\mathfrak{g}}
$$

By straightforward computations, we obtain that Equations (28) and (29) hold for $\rho^{\prime}, \omega^{*}$. For all $x, y, z \in \mathfrak{g}$, we have

$$
\begin{aligned}
& \left(\mathrm{d}_{\rho^{\prime}} \omega^{*}-\mathrm{d}_{\rho} \omega\right)(x, y, z) \\
= & \left(\rho_{\phi_{\mathfrak{g}}(x)}^{\prime} \circ \omega^{*}(y, z)-\omega^{*}\left([x, y]_{\mathfrak{g}}, \phi_{\mathfrak{g}}(z)\right)-\left(\rho_{\phi_{\mathfrak{g}}(x)} \circ \omega(y, z)-\omega\left([x, y]_{\mathfrak{g}}, \phi_{\mathfrak{g}}(z)\right)\right)+c . p .\right. \\
= & A(x, y, z)+A(y, z, x)+A(z, x, y)+B(x, y, z)+B(y, z, x)+B(z, x, y)+C(x, y, z)
\end{aligned}
$$

where

$$
\begin{aligned}
A(x, y, z)= & \rho_{\phi_{\mathfrak{g}}(x)} \circ \rho_{y}(b(z))-\rho_{\phi_{\mathfrak{g}}(y)} \circ \rho_{x}(b(z))-\rho_{[x, y]_{\mathfrak{g}}}\left(b\left(\phi_{\mathfrak{g}}(z)\right)\right)+\left[b\left(\phi_{\mathfrak{g}}(z)\right), \omega(x, y)\right]_{\mathfrak{h}}, \\
B(x, y, z)= & \rho_{\phi_{\mathfrak{g}}(x)}\left([b(y), b(z)]_{\mathfrak{h}}\right)-\left[\rho_{x}(b(y)), b\left(\phi_{\mathfrak{g}}(z)\right)\right]_{\mathfrak{h}}-\left[b\left(\phi_{\mathfrak{g}}(y)\right), \rho_{x}(b(z))\right]_{\mathfrak{h}}, \\
C(x, y, z)= & {\left[b\left(\phi_{\mathfrak{g}}(x)\right),[b(y), b(z)]_{\mathfrak{h}}\right]_{\mathfrak{h}}+\left[b\left(\phi_{\mathfrak{g}}(y)\right),[b(z), b(x)]_{\mathfrak{h}}\right]_{\mathfrak{h}}+\left[b\left(\phi_{\mathfrak{g}}(z)\right),[b(x), b(y)]_{\mathfrak{h}}\right]_{\mathfrak{h}} } \\
& +b\left(\left[[x, y]_{\mathfrak{g}}, \phi_{\mathfrak{g}}(z)\right]_{\mathfrak{g}}\right)+b\left(\left[[y, z]_{\mathfrak{g}}, \phi_{\mathfrak{g}}(x)\right]_{\mathfrak{g}}\right)+b\left(\left[[z, x]_{\mathfrak{g}}, \phi_{\mathfrak{g}}(y)\right]_{\mathfrak{g}}\right)
\end{aligned}
$$

By Equations (27) and (28), we have $A(x, y, z)=0$. Because $\rho_{\phi_{\mathfrak{g}}(x)}$ is a derivation, we obtain $B(x, y, z)=0$. Because $b \circ \phi_{\mathfrak{g}}=\phi_{\mathfrak{h}} \circ b$ and $\mathfrak{g}, \mathfrak{h}$ are Hom-Lie algebras, we obtain $C(x, y, z)=0$. Thus, we have $\mathrm{d}_{\rho} \omega=\mathrm{d}_{\rho^{\prime}} \omega^{*}$. Because Equations (28) and (29) hold for $\rho^{\prime}, \omega^{*}$ and $\rho^{\prime}, \omega^{\prime}$, respectively, we have

$$
\left[\rho_{x}^{\prime}, \rho_{y}^{\prime}\right]_{\phi_{\mathfrak{h}}}-\rho_{[x, y]_{\mathfrak{g}}}^{\prime}=\operatorname{ad}_{\omega^{\prime}(x, y)}=\operatorname{ad}_{\omega^{*}(x, y)}
$$

Thus, we have $\operatorname{ad}_{\left(\omega^{\prime}-\omega^{*}\right)(x, y)}=0$. Moreover, we have $\left(\omega^{\prime}-\omega^{*}\right)(x, y) \in \operatorname{Cen}(\mathfrak{h})$. By Equation (29), we can

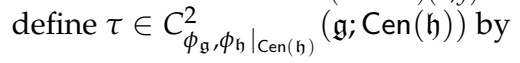

$$
\tau(x, y)=\omega^{\prime}(x, y)-\omega^{*}(x, y)
$$

Thus, we have

$$
\mathrm{d}_{\rho^{\prime}} \omega^{\prime}-\mathrm{d}_{\rho} \omega=\mathrm{d}_{\rho^{\prime}} \omega^{\prime}-\mathrm{d}_{\rho^{\prime}} \omega^{*}=\mathrm{d}_{\rho^{\prime}}\left(\omega^{\prime}-\omega^{*}\right)=\mathrm{d}_{\rho^{\prime}} \tau=\mathrm{d}_{\hat{\rho}} \tau
$$

Therefore, we obtain $\left[\mathrm{d}_{\rho} \omega\right]=\left[\mathrm{d}_{\rho^{\prime}} \omega^{\prime}\right]$. The proof is finished.

Now we are ready to give the main result in this paper, namely, that the obstruction of a Hom-Lie algebra homomorphism $\bar{\rho}: \mathfrak{g} \rightarrow$ Out $(\mathfrak{h})$ being extensible is given by the cohomology class $\left[\mathrm{d}_{\rho} \omega\right] \in \mathcal{H}^{3}(\mathfrak{g} ; \hat{\rho})$.

Theorem 3. Let $\bar{\rho}: \mathfrak{g} \rightarrow$ Out $(\mathfrak{h})$ be a Hom-Lie algebra morphism. Then $\bar{\rho}$ is an extensible homomorphism if and only if

$$
\left[\mathrm{d}_{\rho} \omega\right]=[0]
$$

Proof. Let $\bar{\rho}: \mathfrak{g} \rightarrow$ Out $(\mathfrak{h})$ be an extensible Hom-Lie algebra morphism. Then we can choose a diagonal section $s$ of $\pi: \operatorname{Der}(\mathfrak{h}) \rightarrow \operatorname{Out}(\mathfrak{h})$ and define $\rho$ by Equation (26). Moreover, we can choose a linear map $\omega: \mathfrak{g} \wedge \mathfrak{g} \rightarrow \mathfrak{h}$ such that Equations (27) and (28) hold. Because $\bar{\rho}$ is extensible, we have $\mathrm{d}_{\rho} \omega=0$, which implies that $\left[\mathrm{d}_{\rho} \omega\right]=[0]$.

Conversely, if $\left[\mathrm{d}_{\rho} \omega\right]=[0]$, then there exists $\sigma \in C_{\phi_{\mathfrak{g}}, \phi_{\mathfrak{h}} \mid \operatorname{Cen}(\mathfrak{h})}(\mathfrak{g} ; \operatorname{Cen}(\mathfrak{h}))$ such that $\mathrm{d}_{\rho} \omega=\mathrm{d}_{\hat{\rho}} \sigma$. Thus, we have

$$
\mathrm{d}_{\rho}(\omega-\sigma)=\mathrm{d}_{\rho} \omega-\mathrm{d}_{\rho} \sigma=\mathrm{d}_{\rho} \omega-\mathrm{d}_{\hat{\rho}} \sigma=0
$$

Because $\sigma \in C_{\phi_{\mathfrak{g}},\left.\phi_{\mathfrak{h}}\right|_{\text {Cen (h) }}}^{2}(\mathfrak{g} ; \operatorname{Cen}(\mathfrak{h}))$, we also have

$$
\begin{aligned}
\phi_{\mathfrak{h}} \circ(\omega-\sigma) & =(\omega-\sigma) \circ \phi_{\mathfrak{g}}^{\otimes 2} \\
{\left[\rho_{x}, \rho_{y}\right]_{\phi_{\mathfrak{h}}}-\rho_{[x, y]_{\mathfrak{g}}} } & =\operatorname{ad}_{(\omega-\sigma)(x, y)}
\end{aligned}
$$


By Proposition 1, we can construct a Hom-Lie algebra $\left(\mathfrak{g} \oplus \mathfrak{h},[\cdot, \cdot]_{(\rho, \omega-\sigma)}, \phi\right)$. Therefore, $\bar{\rho}$ is an extensible morphism. The proof is finished.

The following theorem classifies diagonal non-abelian extensions of $\mathfrak{g}$ by $\mathfrak{h}$ once they exist.

Theorem 4. Let $\bar{\rho}: \mathfrak{g} \rightarrow$ Out $(\mathfrak{h})$ be an extensible morphism. Then isomorphism classes of diagonal non-abelian extensions of $\mathfrak{g}$ by $\mathfrak{h}$ induced by $\bar{\rho}$ are parameterized by $\mathcal{H}^{2}(\mathfrak{g} ; \hat{\rho})$.

Proof. Because $\bar{\rho}$ is an extensible morphism, we can choose a diagonal section $s$ of $\pi$ and define $\rho$ by Equation (26). We choose a linear map $\omega$ such that Equations (27) and (28) hold and $\mathrm{d}_{\rho} \omega=0$. Thus, the Hom-Lie algebra $\left(\mathfrak{g} \oplus \mathfrak{h},[\cdot, \cdot]_{(\rho, \omega)}, \phi\right)$ defined by Equations (15) and (16) is a diagonal non-abelian extension of $\mathfrak{g}$ by $\mathfrak{h}$, which is induced by $\bar{\rho}$. Let $s^{\prime}$ be another diagonal section of $\pi$ and define $\rho^{\prime}$ by Equation (26). We also choose a linear map $\omega^{\prime}$ such that Equations (27) and (28) hold and $\mathrm{d}_{\rho^{\prime}} \omega^{\prime}=0$. Because $s$ and $s^{\prime}$ are diagonal sections of $\pi$, there exists a linear map $b: \mathfrak{g} \rightarrow \mathfrak{h}$ such that

$$
\phi_{\mathfrak{h}} \circ b=b \circ \phi_{\mathfrak{g}}, \quad \rho_{x}=\rho_{x}^{\prime}+\operatorname{ad}_{b(x)}
$$

We define $\omega^{*}$ by

$$
\omega^{*}(x, y)=\omega^{\prime}(x, y)+\rho_{x}^{\prime}(b(y))-\rho_{y}^{\prime}(b(x))+[b(x), b(y)]_{\mathfrak{h}}-b[x, y]_{\mathfrak{g}}
$$

By the computation in Lemma 3 , we have $\mathrm{d}_{\rho} \omega^{*}=\mathrm{d}_{\rho^{\prime}} \omega^{\prime}=0$. Thus, the Hom-Lie algebra $\left(\mathfrak{g} \oplus \mathfrak{h},[\cdot, \cdot]_{\left(\rho, \omega^{*}\right)}, \phi\right)$ constructed from $\rho, \omega^{*}$ is isomorphic to the Hom-Lie algebra $\left(\mathfrak{g} \oplus \mathfrak{h},[\cdot, \cdot]_{\left(\rho^{\prime}, \omega,\right)}, \phi\right)$ constructed from $\rho^{\prime}, \omega^{\prime}$. Thus, we only need to study the Hom-Lie algebras constructed from a fixed diagonal section $s$. For all $\tilde{\omega}$, which satisfy Equations (28) and (29) and $\mathrm{d}_{\rho} \tilde{\omega}=0$, we define

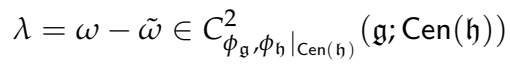

Moreover, we have

$$
\mathrm{d}_{\hat{\rho}} \lambda=\mathrm{d}_{\rho}(\omega-\tilde{\omega})=0-0=0
$$

which implies that $\omega-\tilde{\omega} \in \mathcal{Z}^{2}(\mathfrak{g} ; \hat{\rho})$.

Moreover, let us assume that the Hom-Lie algebra $\left(\mathfrak{g} \oplus \mathfrak{h},[\cdot, \cdot]_{(\rho, \omega)}, \phi\right)$ constructed from $\rho, \omega$ is isomorphic to the Hom-Lie algebra $\left(\mathfrak{g} \oplus \mathfrak{h},[\cdot, \cdot]_{(\rho, \tilde{\omega})}, \phi\right)$ constructed from $\rho, \tilde{\omega}$. Then there exists a linear map $b: \mathfrak{g} \rightarrow \mathfrak{h}$ that does not change $\rho$, that is, $b: \mathfrak{g} \rightarrow \operatorname{Cen}(\mathfrak{h})$, such that

$$
\begin{aligned}
\phi_{\mathfrak{h}} \circ b & =b \circ \phi_{\mathfrak{g}} \\
\omega-\tilde{\omega} & =\mathrm{d}_{\rho} b
\end{aligned}
$$

This is equivalent to $\omega-\tilde{\omega} \in \mathcal{B}^{2}(\mathfrak{g} ; \hat{\rho})$. Thus, isomorphism classes of diagonal non-abelian extensions of $\mathfrak{g}$ by $\mathfrak{h}$ induced by $\bar{\rho}$ are parameterized by $\mathcal{H}^{2}(\mathfrak{g} ; \hat{\rho})$.

Corollary 1. The isomorphism classes of diagonal non-abelian extensions of a Hom-Lie algebra $\mathfrak{g}$ by a Hom-Lie algebra $\mathfrak{h}$ correspond bijectively to the set of pairs $(\bar{\rho},[\kappa])$, where $\bar{\rho}$ is an extensible morphism from $\mathfrak{g}$ to Out $(\mathfrak{h})$ and $[\kappa] \in \mathcal{H}^{2}(\mathfrak{g} ; \hat{\rho})$.

\section{Conclusions}

In this paper, we use a cohomological approach to study diagonal non-abelian extensions of regular Hom-Lie algebras. First, for the case that $\operatorname{Cen}(\mathfrak{h})=0$, we classify diagonal non-abelian extensions of a regular Hom-Lie algebra $\mathfrak{g}$ by a regular Hom-Lie algebra $\mathfrak{h}$ by Hom-Lie algebra morphisms from $\mathfrak{g}$ to the outer derivation of the Hom-Lie algebra Out $(\mathfrak{h})$. More precisely, we show that under the condition $\operatorname{Cen}(\mathfrak{h})=0$, isomorphism classes of diagonal non-abelian extensions of a regular Hom-Lie algebra $\mathfrak{g}$ by a regular Hom-Lie algebra $\mathfrak{h}$ one-to-one correspond to Hom-Lie algebra morphisms from $\mathfrak{g}$ to Out $(\mathfrak{h})$. Then for the general case, isomorphic diagonal non-abelian extensions of a regular Hom-Lie algebra $\mathfrak{g}$ by $\mathfrak{h}$ give rise to the same morphism from $\mathfrak{g}$ to Out $(\mathfrak{h})$. However, given a morphism from $\mathfrak{g}$ to Out $(\mathfrak{h})$, there is an obstruction for the existence of a diagonal non-abelian extension of regular Hom-Lie algebras that induces the given morphism. We show that the obstruction is given by a cohomological class in the third cohomology group. More precisely, if the cohomological class is trivial, then there is a diagonal non-abelian extension of regular Hom-Lie algebras inducing the given morphism. In this case, we say that the given morphism is extensible. In particular, if the third cohomology group is trivial, then 
every Hom-Lie algebra morphism from $\mathfrak{g}$ to Out $(\mathfrak{h})$ is extensible. Moreover, we show that isomorphism classes of diagonal non-abelian extensions of $\mathfrak{g}$ by $\mathfrak{h}$ inducing the given morphism are parameterized by the second cohomology group.

Author Contributions: Lina Song and Rong Tang did this research together and wrote the paper together.

Conflicts of Interest: The authors declare no conflict of interest.

\section{References}

1. Hartwig, J.; Larsson, D.; Silvestrov, S. Deformations of Lie algebras using $\sigma$-derivations. J. Algebra 2006, 295, 314-361.

2. Cai, L.; Sheng, Y. Purely Hom-Lie bialgebras. Sci. China Math. 2017, Unpublished.

3. Hu, N. $q$-Witt algebras, $q$-Lie algebras, $q$-holomorph structure and representations. Algebra Colloq. 1999, $6,51-70$.

4. Larsson, D.; Silvestrov, S. Quasi-hom-Lie algebras, central extensions and 2-cocycle-like identities. J. Algebra 2005, 288, 321-344.

5. Larsson, D.; Silvestrov, S. Quasi-Lie algebras. Contemp. Math. 2005, 391, 241-248.

6. Ammar, F.; Ejbehi, Z.; Makhlouf, A. Cohomology and Deformations of Hom-algebras. J. Lie Theory 2011, 21, 813-836.

7. Makhlouf, A.; Silvestrov, S. Notes on Formal Deformations of Hom-associative and Hom-Lie Algebras. Forum Math. 2010, 22, 715-739.

8. Sheng, Y. Representations of Hom-Lie algebras. Algebras Represent. Theory 2012, 15, 1081-1098.

9. Casas, J.M.; Insua, M.A.; Pacheco, N. On universal central extensions of Hom-Lie algebras. Hacet. J. Math. Stat. 2015, 44, 277-288.

10. Casas, J.M.; Khmaladze, E.; Rego, N.P. A Non-Abelian Tensor Product of Hom-Lie Algebras. Bull. Malays. Math. Sci. Soc. 2016, doi:10.1007/s40840-016-0352-0.

11. Aragon Perinan, M.J.; Calderon Martin, A.J. The structure of split regular Hom-Poisson algebras. Colloq. Math. 2016, 145, 1-13.

12. Aragon Perinan, M.J.; Calderon Martin, A.J. Split regular Hom-Lie algebras. J. Lie Theory 2015, 25, 875-888.

13. Sheng, Y.; Chen, D. Hom-Lie 2-algebras. J. Algebra 2013, 376, 174-195.

14. Song, L.; Tang, R. Derivation Hom-Lie 2-algebras and non-abelian extensions of regular Hom-Lie algebras. J. Algebra Appl. 2018, doi:10.1142/S0219498818500810.

15. Sheng, Y.; Xiong, Z. On hom-Lie algebras. Linear Multilinear Algebra 2015, 63, 2379-2395.

16. Alekseevsky, D.; Michor, P. W.; Ruppert, W. Extensions of Lie algebras. arXiv 2000, arXiv:math.DG/0005042.

17. Alekseevsky, D.; Michor, P. W.; Ruppert, W. Extensions of super Lie algebras. J. Lie Theory 2005, 15, $125-134$.

18. Fregier, Y. Non-abelian cohomology of extensions of Lie algebras as Deligne groupoid. J. Algebra 2014, $398,243-257$.

19. Hegazi, A.S.; Abdelwahab H.; Calderon Martin, A.J. The classification of N-dimensional non-Lie Malcev algebras with (N-4)-dimensional annihilator. Linear Algebra Appl. 2016, 505, 32-56.

20. Hermann, N.K. Non-abelian extensions of topological Lie algebras. Commun. Algebra 2006, 34, 991-1041.

21. Inassaridze, N.; Khmaladze, E.; Ladra, M. Non-abelian cohomology and extensions of Lie algebras. J. Lie Theory 2008, 18, 413-432.

(C) 2017 by the authors. Licensee MDPI, Basel, Switzerland. This article is an open access article distributed under the terms and conditions of the Creative Commons Attribution (CC BY) license (http:/ / creativecommons.org/licenses/by/4.0/). 